\title{
Insatisfação com a imagem corporal e fatores associados em universitários
}

\author{
Cilene Rebolho Martins \\ Universidade Federal de Santa Catarina \\ Alex Pinheiro Gordia \\ Universidade Federal do Recôncavo da Bahia \\ Diego Augusto Santos Silva \\ Educador Físico \\ Teresa Maria Bianchini de Quadros \\ Universidade Federal do Recôncavo da Bahia \\ Elisa Pinheiro Ferrari \\ Universidade do Estado de Santa Catarina \\ Davi Monteiro Teixeira \\ Edio Luiz Petroski \\ Universidade Federal de Santa Catarina
}

\begin{abstract}
Resumo
O objetivo deste estudo foi analisar a insatisfação com a imagem corporal e verificar a associação com o estado nutricional e variáveis sociodemográficas (sexo, faixa etária, turno de estudo e trabalho remunerado) em universitários. Participaram do estudo 865 universitários. A prevalência de insatisfação com a imagem corporal foi de $77,9 \%$ e as variáveis associadas a este desfecho foram sexo e estado nutricional (avaliado pelo IMC com base em dados autorreferidos). Os homens apresentaram mais frequentemente o desejo de aumentar o peso e as mulheres, de reduzir. Universitários com excesso de peso demonstraram maior desejo de reduzir o peso corporal $(\mathrm{RC}=6,83$; IC- $95 \%=3,72-12,54)$, e aqueles com baixo peso apresentaram mais chance de desejar aumentar $(\mathrm{RC}=3,06$; IC-95\% = 1,59-5,88). Destaca-se a importância de promover ações de incentivo à prática de atividades físicas e à adoção de hábitos alimentares saudáveis, bem como a prevenção de comportamentos de risco adotados no sentido de modificara imagem corporal.
\end{abstract}

Palavras-chave: imagem corporal; insatisfação corporal; estado nutricional; universitários.

\begin{abstract}
Body Image dissatisfaction and associated factors among college students. The objective of this study was to analyze the body image dissatisfaction and its association with nutritional status and socio-demographics variables (sex, age group, period of study and paid employment) among college students. The study involved 865 college students. The prevalence of body image dissatisfaction was $77.9 \%$ and sex and nutritional status (assessed by body mass index based on self-reported measures) were associated with this outcome. Men, more often, desired to increase body weight and women, to reduce it. Overweight college students were more likely to desire to reduce body weight $(\mathrm{OR}=6.83 ; 95 \% \mathrm{CI}=3.72-12.54)$, and those who were underweight were more likely to desire to increase it $(\mathrm{OR}=3.06 ; 95 \% \mathrm{CI}=1.59-5.88)$. Thus, it is highlighted the importance of policy to promote physical activity practices and adoption of healthy eating habits, as well as to prevent risk behaviors adopted to change the body image.
\end{abstract}

Keywords: body image; body dissatisfaction; nutritional status; college.

A imagem corporal (IC) é um constructo multidimensional e dinâmico formado pelas imagens ou representações mentais do corpo que se apresentam ao indivíduo no contexto de sua vida, englobando todas as formas pelas quais uma pessoa experiencia e conceitua seu próprio corpo (Tavares, 2003). Dois aspectos específicos da IC podem ser distinguidos: a exatidão da estimativa do tamanho corporal e os sentimentos em relação ao corpo e porções do mesmo (insatisfação corporal ou desvalorização da forma física) (Cordás \& Castilho, 1994). O processo de formação da IC pode ser influenciado pelo sexo, idade, meios de comunicação e pela relação do corpo com os processos cognitivos como crenças, valores e atitudes inseridos 
em uma cultura (Blowers, Loxton, Grady-Flesser, Occhipinti, \& Dawe, 2003; Ricciardelli, McCabe, \& Banfield, 2000).

Normas socioculturais têm perpetuado o estereótipo da associação entre magreza e beleza entre as mulheres, fazendo com que um corpo magro seja considerado ideal. Por outro lado, o padrão de beleza masculino faz com que os homens desejem um corpo maior, mais volumoso e musculoso (Neighbors \& Sobal, 2007). Porém, a impossibilidade de atender aos padrões estéticos pode conduzir a uma IC negativa, a transtornos alimentares, tentativas frustradas de controlar o peso corporal e uso de esteróides anabolizantes (Labre, 2002; Stice \& Shaw, 2002; Stipp \& Oliveira, 2003).

Estudos recentes conduzidos com estudantes universitários nos Estados Unidos têm encontrado forte associação entre IC e índice de massa corporal (IMC), mostrando que indivíduos com valores de IMC correspondentes a sobrepeso e obesidade apresentaram significativamente maior insatisfação, independente do sexo (Gilliard, Lackland, Mountford, \& Egan, 2007; Neighbors \& Sobal, 2007; Watkins, Christie, \& Chally, 2008; Yates, Edman, \& Aruguete, 2004). No Brasil, estudos realizados com universitários são escassos e, além disso, retratam resultados divergentes (Coqueiro, Petroski, Pelegrini, \& Barbosa, 2008; Kakeshita \& Almeida, 2006; Silva, Saenger, \& Pereira, 2011). Por exemplo, constatou-se que universitários da cidade de Ribeirão Preto-SP de ambos os sexos expressaram maior insatisfação com a IC conforme o aumento do IMC (Kakeshita \& Almeida, 2006). Porém, acadêmicos de uma instituição federal do sul do Brasil que apresentaram IMC correspondente a peso normal estavam mais insatisfeitos por excesso de peso do que aqueles que apresentaram sobrepeso e obesidade (Silva et al., 2011). E ainda, estudo realizado com universitários da região de Florianópolis, Santa Catarina, não encontrou associação entre insatisfação com a IC e IMC (Coqueiro et al., 2008).

A associação entre variáveis sociodemográficas (como faixa etária, turno de estudo e trabalho remunerado) e IC em universitários tem sido pouco abordada na literatura. Desta forma, identifica-se a necessidade de analisar estas associações, uma vez que informações desta natureza podem ajudar a identificar o perfil dos acadêmicos que apresentam maior insatisfação com a IC. A partir destes achados, intervenções mais efetivas e eficientes podem ser sugeridas com intuito de reduzir a insatisfação com a IC neste grupo populacional. Neste sentido, o presente estudo teve como objetivo analisar a insatisfação com a IC em estudantes de uma universidade pública brasileira e verificar a associação com o estado nutricional (avaliado por meio do IMC) e com variáveis sociodemográficas.

\section{Método}

Trata-se de um estudo de corte transversal, realizado com base no banco de dados do projeto de pesquisa "Avaliação da aptidão física relacionada à saúde de universitários da Universidade Federal de Santa Catarina (UFSC)". O protocolo do estudo foi aprovado pelo Comitê de Ética em Pesquisa da UFSC (Processo n ${ }^{\circ}$ 096/2007).
A população do estudo foi composta por 2290 universitários ingressantes em uma universidade pública de Florianópolis, Santa Catarina, no primeiro semestre do ano letivo de 2008. Para o cálculo amostral, foi utilizada a metodologia recomendada por Thomas, Nelson e Silverman (2007), considerando-se um nível de confiança igual a $95 \%$ e um erro máximo permitido de 3,0 pontos percentuais. Foi utilizada a amostragem estratificada proporcional por centro de ensino e turno de estudo. Para o sorteio das turmas dentro de cada centro de ensino, utilizou-se o procedimento randomizado.

Com base no cálculo amostral, estimou-se que seria necessário avaliar 728 universitários, no entanto, devido à coleta de dados ter sido realizada por conglomerado de turmas, foram convidados a participar do estudo todos os estudantes presentes em sala de aula no dia da coleta. Portanto, fizeram parte da pesquisa 921 indivíduos. Foram excluídos os universitários com dados incompletos $(n=56)$. A amostra final foi composta de 865 estudantes universitários (498 do sexo masculino e 367 do sexo feminino) com média de 20,5 anos de idade $(D P=5,2)$.

\section{Instrumentos e procedimentos}

A coleta de dados foi realizada no horário de aula dos universitários por uma equipe devidamente treinada para a aplicação dos instrumentos. Para participar da pesquisa, os estudantes assinaram o Termo de Consentimento Livre e Esclarecido.

Para a avaliação da IC, utilizou-se a escala de silhuetas de Stunkard, Sorensene Schulsinger (1983), que é composta por um conjunto de figuras humanas numeradas de 1 a 9 , para ambos os sexos, que representam desde a magreza (silhueta 1) até a obesidade severa (silhueta 9). Foi solicitado aos estudantes que indicassem a figura que melhor representava a sua aparência física atual (silhueta atual), e a que eles gostariam de ter (silhueta desejada). A insatisfação com a IC foi identificada, subtraindose o valor da silhueta atual do valor correspondente à silhueta desejada (silhueta atual-silhueta desejada). Os indivíduos que apresentaram valores positivos foram classificados como insatisfeitos por excesso de peso; os que apresentaram valores negativos foram classificados como insatisfeitos por magreza $\mathrm{e}$ aqueles que apresentaram valor igual a zero foram classificados como satisfeitos.

O estado nutricional foi avaliado mediante o IMC, calculado pelo peso corporal dividido pela estatura ao quadrado $(\mathrm{kg} /$ $\mathrm{m} 2$ ), sendo utilizadas medidas autorreferidas. A validade e fidedignidade destas medidas têm sido demonstradas em estudos prévios (Peixoto, Benício, \& Jardim, 2006; Coqueiro, Borges, Araújo, Pelegrini, \& Barbosa, 2009). Os estudantes foram classificados como baixo peso (IMC $\leq 18,5$ ), peso normal (IMC entre 18,6 e $\leq 24,9)$ e excesso de peso (IMC $\geq 25$ ) (sobrepeso + obesidade), de acordo com os pontos de corte estabelecidos pela Organização Mundial da Saúde (OMS, 1998). As variáveis sociodemográficas analisadas foram sexo, faixa etária $(<20$ e $\geq 20$ anos), trabalho remunerado (sim e não) e turno de estudo (diurno e noturno).

\section{Análise estatística}

A estatística descritiva foi utilizada por meio da distribuição 
de frequências. A análise de regressão logística multinomial foi utilizada para verificar a associação da insatisfação com a IC (variável desfecho) com as variáveis independentes (IMC, sexo, faixa etária, trabalho remunerado e turno de estudo). Optou-se por esta análise em virtude do desfecho apresentar mais do que duas categorias (satisfeito, insatisfeito por excesso e insatisfeito por magreza), utilizando-se os indivíduos satisfeitos como categoria de referência.Os dados foram analisados no programa estatístico SPSS 15.0, adotando-se $p<0,05$ para significância estatística.

\section{Resultados}

$\mathrm{Na}$ Tabela 1estão descritas as características da amostra estudada. A prevalência de insatisfação com a IC foi de $77,9 \%$, sendo maior a proporção de universitários insatisfeitos por excesso de peso que por baixo peso (Tabela 1).

Ao analisar a insatisfação com a IC por sexo, verificouse que, entre as mulheres, houve uma maior prevalência de insatisfação com a IC em relação ao excesso de peso $(62,4 \%)$ do que pela magreza $(15,5 \%)$. Entretanto, no sexo masculino,

Tabela 1

Características da amostra de estudantes universitários ( $n=865)$. Florianópolis, Santa Catarina, 2008.

\begin{tabular}{lrr}
\hline Variáveis & $n$ & \% \\
\hline Sexo & 498 & 57,6 \\
Masculino & 367 & 42,4 \\
Feminino & 533 & 61,6 \\
Faixa etária & 332 & 38,4 \\
$<20$ anos & & 72,1 \\
$\geq 20$ anos & 624 & 27,9 \\
Turno de estudo & 241 & \\
Diurno & & 74,2 \\
Noturno & 642 & 25,8 \\
Trabalho remunerado & 223 \\
Não & & 8,0 \\
Sim & 69 & 76,9 \\
Estado nutricional & 665 & 15,1 \\
Baixo peso & 131 & 22,1 \\
Peso normal & & 46,1 \\
Excesso de peso & 191 & 31,8 \\
Smagem corporal & 399 & 275 \\
Insatisfeito por excesso & & \\
Insatisfeito por magreza & & \\
\hline
\end{tabular}

identificou-se uma maior prevalência de insatisfação com a IC em relação à magreza $(43,8 \%)$ do que pelo excesso de peso $(34,1 \%)$.

As variáveis que apresentaram associação significativa com a insatisfação com a IC foram sexo e IMC (Tabela 2). Em relação ao sexo, os homens apresentaram 4,46 (IC-95\%: $2,78-7,16)$ vezes mais chance de estarem insatisfeitos com a magreza em relação às mulheres. Observou-se, também, que ser estudante do sexo masculino representou menor chance de insatisfação pelo excesso de peso $(\mathrm{RC}=0,30$; IC-95\%: 0,20$0,45)$ quando comparado ao sexo feminino, demonstrando uma tendência das mulheres estarem mais insatisfeitas pelo excesso de peso do que os homens. Quanto ao IMC, os estudantes com baixo peso apresentaram mais chance de estarem insatisfeitos com a IC em relação à magreza $(\mathrm{RC}=3,06$; IC-95\%: 1,59$5,88)$ em comparação àqueles que apresentaram peso normal. Universitários com excesso de peso apresentaram 6,83 (IC-95\%: $3,72-12,54)$ vezes mais chance de estarem insatisfeitos com a IC por excesso em relação aos com peso normal.

As variáveis faixa etária, turno de estudo e trabalho remunerado não apresentaram associação com a insatisfação com a IC.
Os resultados encontrados na amostra de estudantes recém-ingressos em uma universidade pública da cidade de Florianópolis/SC, mostraram que a prevalência de insatisfação com a IC foi elevada $(77,9 \%)$. Outros estudos realizados no Brasil têm descrito resultados semelhantes, sendo que a prevalência em universitários foi de 78,8\% (Coqueiro et al., 2008 ) e de $76 \%$ e $82 \%$ em praticantes de caminhada do sexo feminino e masculino, respectivamente (Damasceno, Lima, Vianna, Vianna, \& Novaes, 2005).

No que diz respeito à insatisfação corporal no sexo feminino, os achados do presente estudo corroboraram outras pesquisas realizadas com universitárias (Cachelin, Rebeck, Chung, \& Pelayo, 2002; Coqueiro et al., 2008; Miller \& Halberstadt, 2005; Gilliard et al., 2007; Neighbors \& Sobal, 2007; Yates et al., 2004). $\mathrm{O}$ fato de mulheres desejarem perder peso pode estar relacionado à influência da mídia e de outros aspectos socioculturais sobre o padrão de beleza feminino (Jones, 2001; Lavine, Sweeney, \& Wagner, 1999; Taquiet al., 2008), pois a supervalorização das formas corporais magras faz com que as mulheres idealizem este padrão. Porém, este corpo desejado nem sempre é alcançado, podendo ocasionar insatisfação e uma autoavaliação negativa do próprio corpo. 
Tabela 2

Regressão multinomial da insatisfação com a imagem corporal e as variáveis independentes (categoria de referência: satisfeito) em universitários. Florianópolis, Santa Catarina, 2008.

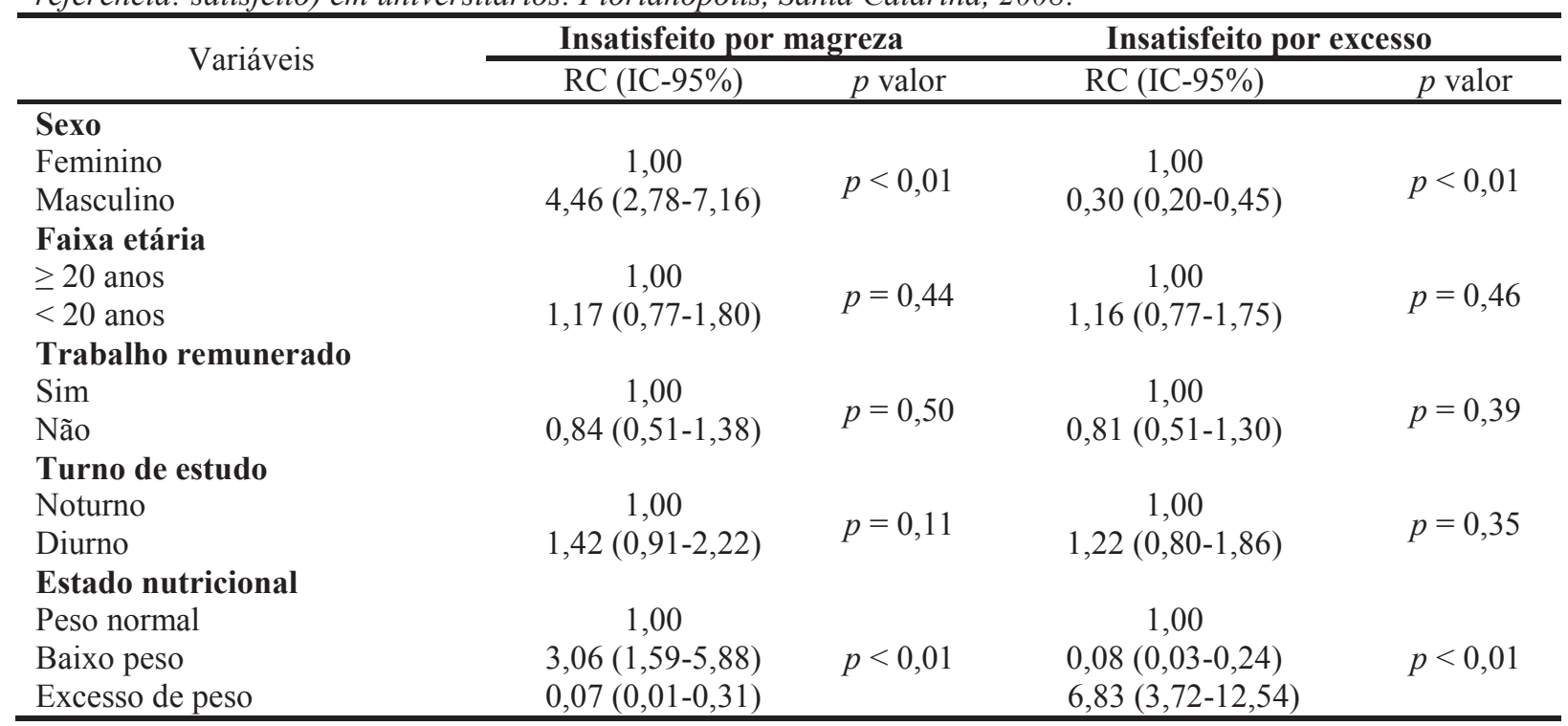

Nota: RC: razão de chance; IC: intervalo de confiança.

Ao analisar a IC no sexo masculino, percebe-se uma divergência entre os resultados retratados na literatura. Algumas investigações constataram que homens, em geral,tendem a estar satisfeitos com a sua IC (Gilliard et al., 2007; Miller \& Halberstadt, 2005). Entretanto, outras pesquisas têm relatado que estes estão insatisfeitos com a IC, ora apresentando o desejo de aumentar o peso (Neigbors \& Sobal, 2007; Cachelin et al., 2002), ora de perder peso(Coqueiro et al., 2008; Pope et al., 2000).

As razões para essas divergências ainda não estão claras. No entanto, sabe-se que os homens também sofrem com a concepção de beleza imposta pela mídia, que, nos tempos atuais, tem exaltado uma musculatura bem desenvolvida (Jones, 2001; Labre, 2002; Lavine et al., 1999; Taqui et al., 2008).Por este motivo, é possível afirmarquea insatisfação em relação à magreza parece estar aumentando entre os homens (Labre, 2002), fato também verificado no presente estudo. Desta forma, o desejo dos homens em querer aumentar o peso pode estar relacionado ao aumento da massa muscular, no sentido de atingir um ideal de atratividade masculina.

No presente estudo, os universitários com baixo peso (ambos os sexos) apresentaram maior insatisfação com a IC em relação aos com peso normal, demonstrando o desejo de aumentar o peso. Este achado pode estar relacionado ao fato de que a amostra foi composta, em sua maioria, por universitários do sexo masculino, os quais demonstram insatisfação com o status de magreza, almejando aumentar as dimensões corporais no sentido de adquirir um porte atlético. Por outro lado, ao analisar estudantes do sexo feminino com baixo peso de uma universidade americana, Neighbors e Sobal (2007) constataram que a maioria estava satisfeita com a IC, uma vez que este estado nutricional corresponde ao padrão desejado pelas mulheres. Desta forma, universitários com baixo peso que demonstram o desejo de aumentar a massa muscular devem ser orientados à prática de atividades físicas adequadas a este objetivo, destacando-se a importância de estratégias de prevenção do uso de esteroides anabolizantes.

Os universitários com excesso de peso mostraram-se insatisfeitos com a IC, indicando desejo de perder peso, assemelhando-se ao que tem sido encontrado em outros estudos conduzidos nesta população (Neighbors \& Sobal, 2007; Gilliard et al., 2007; Kakeshita \& Almeida, 2006). O descontentamento com o excesso de peso pode ser um fator motivacional para a busca de alternativas para a sua redução, facilitando ações de caráter preventivo de doenças crônico-degenerativas associadas ao excesso de peso. Portanto, é importante que os universitários nesta condição nutricional sejam orientados a adotarem hábitos alimentares saudáveis e sejam encorajados à prática de exercícios físicos regulares. Por outro lado, torna-se relevante, também, a prevenção de comportamentos alimentares inadequados adotados com o objetivo de reduzir o peso corporal (por exemplo: autoindução de vômitos, uso de laxantes, jejum prolongado), uma vez que podem acarretar transtornos alimentares, trazendo sérios prejuízos à saúde.

A insatisfação com a IC nos acadêmicos investigados não esteve associada à faixa etária, ao turno de estudo e ao trabalho remunerado. Não foram encontrados estudos com universitários que incluíssem essas variáveis e a associação com a insatisfação corporal, prejudicando a discussão destes resultados. Sugere-se que estudos com acadêmicos de outras instituições de ensino superior do país verifiquem a relação entre IC e estas variáveis sociodemográficas, a fim de identificar se o achado do presente estudo procede nas diferentes regiões do Brasil.

O presente estudo apresenta algumas limitações, como a utilização da escala de silhuetas de Stunkard et al. (1983). Tais silhuetas são bidimensionais e não permitem a representação do indivíduo como um todo, da distribuição da massa de gordura subcutânea, da massa muscular, bem como de outros aspectos antropométricos importantes na formação da IC. Além disso, a 
escolha da silhueta atual possui um caráter muito subjetivo, não sendo possível controlar aspectos complexos que influenciam na IC, como a influência da família, dos amigos, da mídia ou, em alguns casos, de algumas patologias em que ocorre a distorção da IC, como a anorexia, bulimia e dismorfia muscular. Entretanto, o uso desse instrumento tem recebido uma boa aceitação na literatura, sendo amplamente utilizado em estudos nacionais (Coqueiro et al., 2008; Damasceno et al., 2005) e internacionais (Neighbors \& Sobal, 2007; Gilliard et al., 2007, Cachelin et al., 2002), facilitando a comparação dos resultados.Desta forma, o presente estudo vem contribuir com a literatura, sendo o primeiro a analisar a associação da insatisfação com a IC com o estado nutricional e com variáveis sociodemográficas em uma amostra representativa de estudantes de uma universidade pública brasileira.

\section{Conclusões}

Esse estudo permitiu concluir que a prevalência de insatisfação com a IC nos universitários foi elevada, apresentando associação com o sexo e com o estado nutricional.Os homens apresentaram mais frequentemente o desejo de aumentar as dimensões corporais, e as mulheres, de diminuir. No que diz respeito ao IMC, estar com baixo peso ou com excesso de peso gera insatisfação com a IC, e é mais predominante nos indivíduos com excesso de peso.

Os achados do presente estudo reforçam a ideia de que universitários de ambos os sexos e com diferentes classificações de IMC almejam atingir o padrão de beleza determinado por fatores socioculturais. Desta forma, as questões levantadas devem servir de alerta para a prevenção de transtornos alimentares, uso de esteroides anabolizantes e prática excessiva de exercício físico em estudantes universitários. Além disso, destaca-se a importância de promover ações que visem à adoção de prática regular de atividades físicas e de hábitos alimentares saudáveis, a fim de promover uma maior satisfação com a IC nesse segmento da população.

\section{Referências}

Blowers, L. C., Loxton, N. J., Grady-Flesser, M. G., Occhipinti, S., \& Dawe, S. (2003). The relationship between sociocultural pressure to be thin and body dissatisfaction in preadolescent girls. Eating Behaviors, 4(3), 229-244.

Cachelin, F. M., Rebeck, R. M., Chung, G. H., \& Pelayo, E. (2002). Does ethnicity influence body-size preference? A comparison of body image and body size. Obesity Research, 10(3), 158-166.

Coqueiro, R. S., Borges, L. J., Araújo, V. C., Pelegrini, A., \& Barbosa, A. R. (2009). Medidas auto-referidas são válidas para avaliação do estado nutricional na população brasileira? Revista Brasileira de Cineantropometria e Desempenho Humano, 11(1), 113-119.

Coqueiro, R. S., Petroski, E. L., Pelegrini, A., \& Barbosa, A. R. (2008). Insatisfação com a imagem corporal: avaliação comparativa da associação com estado nutricional em universitários. Revista de Psiquiatria do Rio Grande do Sul, 30(1), 31-38.

Cordás, T. A., \& Castilho, S. (1994). Imagem corporal nos transtornos alimentares -Instrumento de avaliação: "Body shape questionnaire". Psiquiatria Biológica, 2(1), 17-21.
Damasceno, V. O., Lima, J. R. P., Vianna, J. M., Vianna, V. R. A., \& Novaes, J. S. (2005). Tipo físico ideal e satisfação com a imagem corporal de praticantes de caminhada. Revista Brasileira de Medicina do Esporte, 11(3), 181-186.

Gilliard, T.S., Lackland, D. T., Mountford, W. K., \& Egan, B. M. (2007). Concordance between self-reported heights and weights and current and ideal body images in young adult African American men and women. Ethnicity \& Disease, 17(4), 617-623.

Jones, D.C. (2001). Social comparison and body image: attractiveness comparisons to models and peers among adolescent girls and boys. Sex Roles, 45(9/10), 645-664.

Kakeshita, I. S., \&Almeida, S. S. (2006). Relação entre índice de massa corporal e a percepção da auto-imagem em universitários. Revista de Saúde Pública, 40(3), 497-504.

Labre, M. P. (2002). Adolescent boys and the muscular male body ideal. Journal of Adolescent Health, 30(4), 233-242.

Lavine, H., Sweeney, D., \& Wagner, S.H. (1999). Depicting women as sex objects intelevision advertising: effects on body dissatisfaction. Personality and Social Psychology Bulletin, 25(8), 1049-1058.

Miller, E., \& Halberstadt, J. (2005). Media consumption, body image and thin ideals in New Zealand men and women. New Zealand Journal of Psychology, 34(3), 189-195.

Neighbors, L. A., \& Sobal, J. (2007). Prevalence and magnitude of body weight and shape dissatisfaction among university students. Eating Behaviors, $8(4), 429-439$.

Peixoto, M. R. G., Benício, M. H. D., \& Jardim, P. C. B. V. (2006). Validade do peso e da altura auto-referidos: o estudo de Goiânia. Revista de Saúde Pública, 40(6), 1065-1072.

Pope, H. G., Gruber, A. J., Mangweth, B., Bureau, B., deCol, C., Jouvent. R., ... Hudson, J. I. (2000). Body image perception among men in three countries. American Journal of Psychiatry, 157(8), 1297-1301.

Ricciardelli, L. A., McCabe, M. P., \& Banfield, S. (2000). Body image and body change methods in adolescent boys. Role of parents, friends, and the media. Journal of Psychosomatic Research, 49(3), 189-197.

Silva, T. R., Saenger, G., \& Pereira, E. F. (2011). Fatores associados à imagem corporal em estudantes de Educação Física. Motriz, 17(4), 630-639.

Stice, E., \& Shaw, H. E. (2002). Role of body dissatisfaction in the onset and maintenance of eating pathology: a synthesis of research findings. Journal of Psychosomatic Research, 53(5), 985-993.

Stipp, L. M., \& Oliveira, M. R. M. (2003). Imagem corporal e atitudes alimentares: diferenças entre estudantes de nutrição e de psicologia. Saúde em Revista, 5(9), 47-51.

Stunkard, A. J., Sorensen, T., \& Schulsinger, F. (1983).Use of the danish adoption register for the study of obesity and thinness. In S. S. Kety, L. P. Rowland, E. L.Sidman \& S. W. Matthysse (Orgs.), The Genetics of Neurological and Psychiatric Disorders (pp. 115-120). Nova Iorque: Raven Press.

Taqui, A. M., Shaikh, M., Gowani, S. A., Shahid, F., Khan, A., Tayyeb, S. M.,...Naquiv. H. A. (2008). Dysmorphic disorder: gender differences and prevalence in a Pakistani medical student population. BMC Psychiatry, 8, 20. doi: 10.1590/S0365-05962009000600002

Tavares, M. C. G. C. F. (2003). Imagem corporal - conceito e desenvolvimento. São Paulo: Manole.

Thomas, J. R., Nelson, J. K., \& Silverman, S. J. (2007). Métodos de pesquisa em atividade fisica. $5^{\mathrm{a}}$ ed. Porto Alegre: Artmed.

Watkins, J. A., Christie, C., \& Chally, P. (2008). Relationsip between body image and body mass index in college men. Journal of American College Health, 57(1), 95-100.

World Health Organization (1998). Obesity: preventing and managing the global epidemic. Genebra: World Health Organization.

Yates, A., Edman, J., \& Aruguete, M. (2004).Ethnic differences in BMI and body/ self-dissatisfaction among whites, Asian subgroups, Pacific Islanders, and African-Americans. Journal Adolescent Health, 34(4), 300-307. 
Cilene Rebolho Martins, mestre em Educação Física pela Universidade Federal de Santa Catarina, é doutoranda no Programa de Pós-graduação em Educação Física da Universidade Federal de Santa Catarina. Endereço para correspondência: Universidade Federal de Santa Catarina, Centro de Desportos - Núcleo de Cineantropometria e Desempenho Humano - Campus Universitário - Trindade. Caixa Postal 476 - 88040-900 -Florianópolis, SC. Telefone: (48) 3721-6342. Fax: (48) 3721-8562. E-mail: cilenerebolho@yahoo.com.br Alex Pinheiro Gordia, mestre em Educação Física pela Universidade Federal do Paraná, é professor Assistente na Universidade Federal do Recôncavo da Bahia. E-mail: alexgordia@gmail.com

Diego Augusto Santos Silva é doutor em Educação Física pela Universidade Federal de Santa Catarina. E-mail: diegoaugustoss@yahoo.com.br

Teresa Maria Bianchini de Quadros, mestre em Educação Física pela Universidade Federal de Santa Catarina, é professora Assistente na Universidade Federal do Recôncavo da Bahia. E-mail: tetemb@gmail.com

Elisa Pinheiro Ferrari, mestre em Educação Física pela Universidade Federal de Santa Catarina, é doutoranda no Programa de Pós-graduação em Ciências do Movimento Humano da Universidade do Estado de Santa Catarina.E-mail: elisaferrari_@hotmail.com

Davi Monteiro Teixeira, graduado em Educação Física pela Universidade Federal de Santa Catarina, é professor colaborador do Núcleo de Pesquisa em Cineantropometria e Desempenho Humano da Universidade Federal de Santa Catarina. E-mail: davitrinda@hotmail.com

Edio Luiz Petroski, doutor em Educação Física pela Universidade Federal de Santa Maria e pó-doutor pela Universidade de Montreal e pela Faculdade de Motricidade Humana, é professor Titular da Universidade Federal de Santa Catarina. E-mail: petroski@cds.ufsc.br 